Revista

\title{
Multi-Ensayos
}

Vol. 4, $\mathrm{N}^{\circ} 7$

ISSN: 2412-3285

https://multiensayos.unan.edu.ni

DOI: https://doi.org/10.5377/multiensayos.v4i7.9491

\section{Uso de multimedia en entornos de plataformas virtuales de aprendizajes}

\section{Using Multimedia in Virtual Learning Platform Environments}

\author{
Manuel Rivas Chavarría ${ }^{1}$
}

Recibido: 01 de marzo de 2018, Aceptado: 27 de septiembre de 2018

\section{RESUMEN}

El comunicarnos, compartir conocimientos y experiencias es algo inherente en los seres humanos, las maneras de hacerlo han evolucionado al transcurrir el tiempo. Con este escrito mi deseo es cooperar con otras personas a cerca de algunas prácticas y recursos existentes en las plataformas virtuales de aprendizajes, particularmente la inclusión de multimedia que resultan ser un gran apoyo en el rol de facilitador o tutor virtual que es hacia donde se enrumban los nuevos paradigmas de la educación, es un vasto recurso, que se puede definir como un sistema que utiliza más de un medio de comunicación para transmitir o presentar información, combinando texto, imagen, sonido y vídeo. Además, que se puede usar de modo síncrono (comunicación en tiempo real) o bien de manera asíncrona (comunicación no en tiempo real).

Palabras claves: multimedia; virtualización; plataformas virtuales.

\section{ABSTRACT}

Communicating, sharing knowledge and experiences is something inherent in human beings, the ways of doing it have evolved over time. Through this essay, I desire to share with other people the practices and existing resources in the virtual learning platforms, particularly the inclusion of multimedia, which turns out to be a great support for facilitators and virtual tutors as this is the future of new paradigms of education. Multimedia is a vast resource, which can be defined as a system that uses more than one means of communication to send or present information, combining text, image, sound and video. In addition, it can be used synchronously (communication in real time) or asynchronously (communication not in real time).

Keywords: multimedia; virtualization; virtual platforms.

1 Docente del Departamento de Ciencia, Tecnología y Salud, UNAN-Managua/FAREM-Estelí. Correo electrónico: mrivas@unan.edu.ni Copyright (c) 2018 Revista Multi-Ensayos. 


\section{INTRODUCCIÓN}

La tendencia de la educación se enrumba hacia la virtualización, en ese sentido una herramienta de mucha ayuda es la multimedia como recurso didáctico que se pueden usar aprovechando que es una de las bondades que facilitan los entornos de las plataformas virtuales. Es importante. hacer notar que no es la única herramienta a implementar y que es oportuno decir que se pueden construir o utilizar existentes.

A través de la utilización de este recurso como facilitador, he tenido la oportunidad de motivar a mis estudiantes enfatizándoles que ellos serán profesionales en TIC y que por ende deben aprovechar este gigantesco mundo digital. Como docentes debemos reconocer que los métodos y estrategias para compartir conocimientos han cambiado siendo esto un reto que nos debe motivar a subirnos al tren de las plataformas virtuales para provocar un cambio en nuestro quehacer docente y perderle el miedo para explotar este fenomenal recurso.

\section{DESARROLLO}

Es adecuado crear un entorno de entendimiento para los colegas que no están familiarizados con algunos conceptos, que al ser de uso frecuente y que son inherentes a estos entornos de aprendizajes logramos agregarlos a nuestro léxico casi cotidiano.

Multimedia: es un término que procede de la lengua inglesa y que refiere a aquello que utiliza varios medios de manera simultánea en la transmisión de una información. Una presentación multimedia, por lo tanto, puede incluir fotografías, vídeos, sonidos y texto.

Como lo expresan Pérez y Gardey, (2014), es un recurso de los más completo porque involucra el uso de diversos medios para lograr una comunicación asertiva, puede ser usado las veces que el estudiante lo estime conveniente, reproduciéndolo por completo, haciendo pausa en cualquier momento, retrocediéndolo, adelantándolo, en fin, hasta lograr la comprensión de la información que la multimedia tiene como propósito.

Si implementamos estrategias acertadas con esos medios, logramos sacarle el máximo provecho como recursos didácticos.

Recurso didáctico: distintos elementos que pueden agruparse en un conjunto, reunidos de acuerdo a su utilización en algún fin específico. Los elementos del conjunto pueden ser reales (físicos), virtuales o abstractos.

El material didáctico es aquel que reúne medios y recursos que facilitan la enseñanza y el aprendizaje. Suelen utilizarse dentro del ambiente educativo para facilitar la adquisición de conceptos, habilidades, actitudes y destrezas (Porto, 2008).

Retomando lo que dice (Porto, 2008), al referirse a recursos didácticos donde claramente si leemos las dos definiciones (multimedia y recursos didácticos), percibimos que están estrechamente relacionadas con 
los elementos que tradicionalmente hemos usado en nuestros procesos de facilitación, solamente que se convierten en virtuales, es decir, intangibles pero que nos han acompañado siempre y por lo tanto al ser inmateriales los podemos encontrar como una de las tantas herramientas de las plataformas virtuales de aprendizajes.

Ahora bien, dice (Virtuales, s.f.) que las plataformas virtuales son un software (programa informático) que proporciona la logística necesaria para llevar a cabo la formación on-line.

Es importante destacar que hay numerosas plataformas virtuales, las cuales tiene la característica de ser un software diseñado para ayudar, en el diseño y desarrollo de cursos en internet. Permiten mejorar la comunicación (alumno-docente; alumno-alumno) y desarrollar el aprendizaje individual y colectivo, pero no serán el sustituto definitivo del facilitador.

En estos entornos nos encontramos con diversas herramientas para actividades que son de mucha importancia y utilidad, entre las cuales se mencionan algunas:

Chat: permite a los participantes (profesor y estudiantes) tener una discusión en formato texto de manera sincrónica en tiempo real. También la comunicación y los elementos que en ella intervienen los podemos encontrar de forma asíncrona, es decir, es aquella que se establece entre personas de manera diferida en el tiempo, esto es, cuando no existe coincidencia.

Foros: permite a los participantes tener discusiones asincrónicas, es decir, discusiones que tienen lugar durante un período prolongado de tiempo.

Glosarios: permite a los participantes crear y mantener una lista de definiciones, de forma similar a un diccionario o para recoger y organizar recursos o información.

Existen más herramientas en una plataforma virtual de aprendizajes, pero hice alusión a estas tres porque las considero estáticas, aunque el chat es interactivo, pero no se puede manipular como lo hacemos con un video, lo digo, por las experiencias aprendidas. A la mayoría de los estudiantes les resulta más interesante reproducir un recurso multimedia que puedan ver y escuchar las veces que quieran, a usar una de estas herramientas las cuales se limitan hacia el texto, separando los demás elementos. Ellos pueden descargarlos para ser portados en distintos dispositivos de reproducción siendo esta una de las principales ventajas, así como también combinando la forma on-line a off- line donde el internet deja de ser necesario.

Es importante, decir que como en la mayoría de las cosas, no es bueno abusar en el uso de estos recursos, deben ser bien usados, y esto se debe tener presente cuando estamos planificando y diseñando nuestros cursos, anteriormente comentaba que los recursos multimedia incluidos se pueden construir o utilizar existentes, por supuesto estos deben ser validados antes de su publicación en las plataformas virtuales de aprendizajes.

En ese sentido el experto (Vega, 2014) nos comparte algunos principios para la elaboración de recursos multimedia: 
- Cuanto menos más: evite lo innecesario.

- Lo técnico supeditado a lo didáctico: primero lo didáctico.

- Legibilidad contra irritabilidad: textos claros y fáciles de leer.

- Evite el aburrimiento: que sea atractivo.

- Interactividad: animación y posibilidad de interacción con la máquina y persona.

- Hipertextualidad: html contra texto plano.

- Flexibilidad: posibilidad de variación, evitar la rigidez.

Criterios para la selección de recursos multimedia (usar existentes):

- Los objetivos educativos que pretendemos lograr.

- Los contenidos que se van a tratar utilizando el material, que concuerden con los contenidos de la asignatura.

- Las características de los estudiantes que los utilizarán: capacidades, estilos cognitivos, intereses, conocimientos previos, experiencia y habilidades requeridas para el uso de estos materiales...

- Las características del contexto (físico, curricular...).

- Las estrategias didácticas que podemos diseñar considerando la utilización del material.

Antes de terminar quiero expresar que, se pueden implementar diversas metodologías, estrategias y técnicas en las plataformas virtuales de aprendizajes para compartir conocimientos, solamente es echar a andar tu creatividad.

\section{CONCLUSIONES}

La educación está apostando al uso de plataformas virtuales de aprendizajes, vienen a representar en el presente una gran oportunidad para la educación, ofreciendo grandes beneficios y conllevando a que tengan acceso muchas personas porque trasciende de las cuestiones geográficas y los edificios de los recintos.

Las herramientas multimedia han abonado significativamente a estos nuevos paradigmas de los procesos de enseñanzas-aprendizajes, más aún cuando nos encontramos con el reto de profesionalizar personas que en su mayoría son jóvenes pertenecientes a la era digital, donde nosotros somos migrantes. He ahí el principal reto "debemos ponernos en sintonía con los nuevos tiempos. Nos encontraremos con estudiantes con muchos conocimientos en las TIC, por tanto, se requiere una nueva visión y compromiso de los docentes".

\section{BIBLIOGRAFÍA}

Pérez P. y Gardey. (2014). Definicion.de. Obtenido de https://definicion.de/multimedia/

Porto, J. P. (2008). Definicion.de. Obtenido de https://definicion.de/material-didactico/

Vega, E . A. (16 de agosto de 2014). In.slideshare. Obtenido de https://es.slideshare.net/stbncr/lastecnologas-educativas-ensayo-multimedia

Virtuales, F. O. (s.f.). Sistemas virtuales.com. Obtenido de http://www.feriaonline.com/bits-elearning/ definicion-de-plataforma-virtual.asp 